\title{
Hepatocellular Carcinoma: An Overview of the Changing Landscape of Treatment Options
}

\author{
Andreas Koulouris (D) \\ Christos Tsagkaris $\mathbb{D}^{2}$ \\ Vasiliki Spyrou (iD ${ }^{3}$ \\ Eleni Pappa (iD ${ }^{4}$ \\ Aikaterini Troullinou ${ }^{2}$ \\ Michail Nikolaou (iD) ${ }^{5}$ \\ 'Resident of Medical Oncology, \\ University General Hospital of Heraklion, \\ University of Crete, Crete, Greece; \\ 2Medical School, University of Crete, \\ Crete, Greece; ${ }^{3}$ Hygeia Hospital, Athens, \\ Greece; ${ }^{4}$ Medical School, National and \\ Kapodistrian University of Athens, \\ Athens, Greece; ${ }^{5}$ Ist Oncology \\ Department, "Saint Savas" Anticancer - \\ Oncology Hospital, Athens, Greece
}

\begin{abstract}
The last three years have seen remarkable progress in comprehending predisposing factors and upgrading our treatment arsenal concerning hepatocellular carcinoma (HCC). Until recently, there were no means to withstand the progression of viral hepatitis-associated liver cirrhosis to HCC. A deeper understanding of the molecular mechanism of the disease, the use of biomarkers, and the follow-up, allowed us to realize that conventional chemotherapy failing to increase survival in patients with advanced HCC tends to be exiled from clinical practice. Multi-kinase inhibitors (TKIs) such as sorafenib, lenvatinib targeting mainly the vascular endothelial growth factor receptors 1-3 VEGFRs 1-3 provided until recently the standard of care for these patients, as first- or second-line treatment. Since May 2020, the atezolizumab plus bevacizumab combination (immunotherapy plus antiVEGF) has become the new reference standard in first-line HCC treatment. Additionally, anti-programmed cell death protein 1 (anti-PD-1) immunotherapy can be used as a secondline treatment following first-line treatment's failure. Phase III clinical trials have recently suggested the efficacy of novel anti-angiogenic factors such as cabozantinib and ramucirumab as a second-line treatment option. With considerations about toxicity arising, clinical trials are investigating combinations of the aforementioned targeted therapies with immunotherapy as first-line treatment. This paper aims to perform a systematic review describing the evolving treatment options for HCC over the last decades, ranging from neoadjuvant treatment to systemic therapy of advanced-stage HCC. With the landscape of HCC treatment shifting towards novel agents the forming of a new therapeutic algorithm for HCC seems to be imperative.
\end{abstract}

Keywords: hepatocellular carcinoma, immunotherapy, targeted therapy, tyrosine kinase inhibitors, biomarkers

\section{Introduction}

Hepatocellular carcinoma (HCC) is the sixth most frequently diagnosed ( 5 th in men and 7th in women) and the third most lethal malignancy. Its incidence has more than tripled in the last 30 years (1.5-6.7/100.000), with an average rise of $3 \%$ per year during 2004-2013. ${ }^{1-3}$ HCC, accounts for roughly $85-90 \%$ of primary liver carcinomas worldwide, being the most common histological subtype. Its five-year survival rate is $16.6 \%$. Its incidence in 2020 mounted up to 905,677 new cases. Its incidence rate is 5.6 per 100,000 in Western Europe, 6.9 per 100.000 in America and up to $17 \%$ in Asia. ${ }^{3}$ It usually develops in the setting of liver cirrhosis, owing to chronic hepatic inflammation and fibrosis. ${ }^{4}$ Nevertheless, numerous cases of de novo disease in non-cirrhotic patients have led to debunking the belief that it only develops in cirrhotic livers. There have also been reports of HCC occurring in
Correspondence: Michail Nikolaou Ist Oncology Department, "Saint Savas" Anticancer - Oncology Hospital, Athens, Greece

Email nikolaoumike@hotmail.com 
patients not only lacking signs of cirrhosis but also without histological evidence of hepatitis. ${ }^{5,6}$ However, cirrhosis is still present at the time of diagnosis in the majority of cases. Leading causes of liver damage include viral hepatitis B (HBV) - with or without hepatitis D - and hepatitis $\mathrm{C}(\mathrm{HCV})$, which until recently thought to account for almost $80 \%$ of cases, as well as, alcohol abuse, NonAlcoholic Steatohepatitis (NASH), autoimmune hepatitis, aflatoxins, obesity and diabetes. ${ }^{7}$ The last two consist risk factors for non-alcoholic fatty liver disease (NAFLD). NAFLD is now considered the hepatic manifestation of metabolic syndrome, whose incidence rate has been on the rise. ${ }^{8} \mathrm{HCV}$-related liver damage and, therefore, HCC is expected to drop drastically over the next few years due to the Direct Antiviral Agents (DAAs). The recently discovered DAAs, offer a radical treatment for HCV infection. Thus, a major shift is imminent in the profile of patients at risk for HCC development, with the HCV-related subset being largely replaced by the concurrent rise of NAFLDrelated disease. ${ }^{9,10}$ Constantly emerging data highlight the differences in the pathogenesis and the clinical presentation between NAFLD-related and HCV-related HCC. This suggests the dire need for further research in this particular subset of patients, with NAFLD-related disease. Factors related to disease progression must be determined leading to a potential alteration in the existing protocols. ${ }^{11,12}$

After a diagnosis has been established, staging will determine therapeutic options. As far as the early stages are concerned, the main objective is the cure. Thus, treatment options are mainly surgical including whole or partial hepatectomy and liver transplant when necessary. In cases where surgical excision is not feasible, radiofrequency (RF) or microwave ablation is recommended. ${ }^{13}$ Treatment of choice for intermediate stages subsume chemoembolism, radioembolism or simple embolism. ${ }^{14}$ Until recently, systemic treatment for advanced liver disease remained a grey zone despite the constant developments. It, first, became an option in 2008, when sorafenib, a targeted multi-kinase inhibitor, showed a median survival benefit of 2.8 months compared to placebo. It is an expensive, highly toxic treatment that, nonetheless, became established as it was the sole therapeutic option available to this subset of patients until the discovery of regorafenib, a tyrosine kinase inhibitor, in $2017 .^{15,16}$ Another targeted agent of the same category called lenvatinib was recently approved (August 2018) as a first-line treatment for non-excisable HCCs. ${ }^{17}$ Due to the significant toxicity associated with sorafenib and regorafenib and their lower objective response rates compared to immunotherapy, immune checkpoint inhibitors (ICIs) such as nivolumab and pembrolizumab are recommended as second-line treatments. It is worth noting that phase III trials of immunotherapy regimens did not yield any statistically significant benefit regarding progression-free and overall survival rates only until recently. In May 2020, the IMbrave 150 study showed a survival advantage of atezolizumab plus bevacizumab over other options in the first-line treatment of aHCC patients in a network metaanalysis. The combination of atezolizumab plus bevacizumab improved OS and PFS over sorafenib in the IMbrave 150 trial and became the new reference standard in the first-line HCC treatment. ${ }^{18,19}$ Lastly, two new antiangiogenic factors called cabozantinib and ramucirumab have recently been added as second-line treatment options, further expanding the growing list of therapeutic agents. ${ }^{20}$

The rapidly emerging new data has drastically changed the landscape when it comes to HCC. Diagnosis can now be achieved using non-invasive imaging techniques and there are a plethora of treatment options available. Proper use of emerging treatments remains a challenge, as the current holistic approach to cancer patients raises the need for therapies that contribute not only to the patients' overall survival but to their quality of life as well. The authors aim to provide a comprehensive overview of the emerging treatment options discussing them with regard to recent clinical trials and contemporary clinical practice.

\section{Methodology}

Specific keywords (hepatocellular carcinoma, systematic therapy, cancer, biomarkers, immunotherapy, chemotherapy) were subjected to list down and analyze the literature in PubMed and Google Scholar. All types of articles, including original research, systematic reviews, and meta analyses, were included besides reports regarding neuroinflammation dating back to 2000. We excluded outdated studies and studies reporting a significant conflict of interest. Out of all articles, as per inclusion criteria and readings, we selected 80 for further analysis and narrative to build.

\section{The Emerging Biomarkers}

Despite the variety of biomarkers been validated in other tumor types, including PDL-1 expression, MSI status, and tumor mutational burden, this is not the case as far as HCC is concerned. Unfortunately, the clinical utility of all available biomarkers is limited at this time. A plethora of 
factors such as des- $\gamma$-carboxy prothrombin, glypican-3, cytokeratin 19, Next-Generation Sequencing (NGS), proteomic analysis, circulating microRNAs, enumeration of Circulating Tumor Cells (CTCs) and quantification of cellfree DNA have been tested as potential biomarkers of early diagnosis without yielding satisfactory results. ${ }^{21}$ AFP seems to be the only available evidence-based diagnostic, predictive and prognostic tool. ${ }^{19,22}$ Particularly since May 2019 FDA approval, it consists the only biomarker to guide treatment decisions (ramucirumab in AFP>400ng/mL). ${ }^{19,23}$

The evaluation of liver function unveils another unexpected, both prognostic and predictive biomarker. Maintenance of liver function may widen the "window of opportunity", which plays a crucial role when it comes to the proper management of the disease. ${ }^{24}$ Particularly, it has been demonstrated that the immediate initiation of treatment at the early stages of cirrhosis (Child-Pugh A) is beneficial. The significance of prompt targeting of this narrow window, before the deterioration of the liver function (Child-Pugh score $\geq 7$ ) is illustrated by the studies of approval of lenvatinib, pembrolizumab and ramucirumab, which will be described below. It is noteworthy that the overall survival of the recruited patients was significantly increased, when their Child-Pugh score was 5 instead of 6 or when their Albumin-Bilirubin (ALBI) grade was 1 instead of 2 , to wit at an earlier stage of the disease. ${ }^{25-27}$

As far as immune biomarkers are concerned, the research field of HCC yields encouraging results. In $\mathrm{HCC}$, tumors can be divided into two basic immune categories: non-inflamed "cold" tumors, which represent $70 \%$ of HCCs and the remaining 30\% of inflamed "hot" tumors (active and exhausted immune subtypes included). The former is mostly characterized by Wnt/CTNNB1 mutations and is related to primary resistance to immune checkpoint inhibitors. More particularly, the presence of Wnt/ CTNNB1 mutations was not associated with ProgressionFree Survival (PFS) benefit. On the contrary, the active immune class may form the matrix of the development of new signatures of response to immunotherapy. ${ }^{27,28}$ These two separate categories are also confirmed by a study of HCC samples of 956 participants. One-quarter of them expressed inflammatory markers, which were indicative of adaptive or exhausted immune response. This renders this sub-group an optimal candidate for immunotherapy. ${ }^{29}$ Furthermore, this class of HCC is not associated with $\beta$ catenin activation, which entails resistance to anti-PD1 therapy through promoting immune escape. It is worth mentioning that $\beta$-catenin activation could be a promising novel biomarker when it comes to $\mathrm{HCC}$ patients' exclusion regarding immunotherapy. ${ }^{28,30}$

In addition, linear non-coding RNAs (ncRNAs), such as long ncRNAs and microRNAs, have been found to affect the regulation of tumor immunity and immunotherapy. Unfortunately, the evidence regarding circRNA is still in infant age. A recent study pointed out their interplay with the tumor microenvironment (TME), suggesting TME as an additional therapeutic target. Merging next-generation sequencing technology with machine learning-based algorithms is expected to provide further information regarding the level of involvement of ncRNAs subtypes in HCC progression. ${ }^{31}$ Suffice it to state that all these biomarkers mentioned above - Wnt/beta-catenin, non-coding RNAs and circRNAs - have not been validated so far, and thus are not routinely used in clinical practice.

\section{Neoadjuvant Treatment}

Neoadjuvant therapies are commonly used for patients with solid-organ malignancies other than $\mathrm{HCC}$, to downstage advanced disease and render the patient capable of receiving surgical therapy. Contrariwise, the role of neoadjuvant therapy in the management of HCC has yet to be established. The neoadjuvant approach may be appropriate in some cases, given that $\mathrm{HCC}$ is distinguished for some unique biologic characteristics. Its relatively aggressive nature, frequently delayed diagnosis and the presence of underlying cirrhosis, are characteristics that entail the paramount need to preserve normal liver function at the time of surgery. ${ }^{32}$ TACE combines transarterial embolization (TAE) with chemotherapy infusion. Although some studies suggest that TACE is beneficial to some patients by rendering their tumors resectable and improving their long-term outcomes, this technique currently applies to a minority of patients with HCC. Further evidence is needed to determine which patients will reap its benefit. Its role in the neoadjuvant setting is not well established and it is not routinely recommended. ${ }^{33}$

Transarterial radioembolization (TARE) is increasingly being performed as an alternative to TACE for patients with HCC. Yttrium-90 (Y-90) loaded microspheres are injected, via arterial cannulation of the feeding vessel to the tumor. This method has fewer side effects compared to TACE. Another beneficial feature of this method is the subsequent hypertrophy of the contralateral future liver remnant in addition to its cytotoxic effects on the tumor, 
introducing the concept of radiation lobectomy (RL). ${ }^{34}$ Furthermore, it is noteworthy that it could be applied in cases of portal vein thrombosis in contrast to TACE. TARE with Y-90 may be implicated in the neoadjuvant setting for patients with advanced HCC who require downstaging for resection. ${ }^{35}$ However, further research is needed so that neoadjuvant TARE can be included in the future versions of guidelines.

Finally, neither sorafenib nor any of the immunotherapy options that are indicated for advanced HCC have been approved in the neoadjuvant setting up to date. Clinical trials regarding immune checkpoint inhibitors are ongoing at this time, whereas antiviral therapy before resection should be considered as part of the multidisciplinary treatment of patients with $\mathrm{HBV}$ or HCV-related HCC. However, their use in neoadjuvant treatment seems to be limited and questionable in contrast to their utility in the adjuvant setting. ${ }^{36,37}$

\section{Postoperative Therapy}

As customary in other malignancies, the utilization of adjuvant treatment after hepatectomy serves the purpose of decreasing the incidence of $\mathrm{HCC}$ recurrence due to either postoperative remnant and intrahepatic metastases (early recurrence) or the development of de novo tumors due to underlying liver disease (late recurrence). However, it remains highly controversial whether its effect on $\mathrm{HCC}$ is beneficial. There are a plethora of therapeutic choices that have been tested, such as adjuvant chemotherapy with 5-FU, sorafenib, activated cytokine-induced killer cells and glypican 3 (GPC3) vaccine. ${ }^{37}$

A valuable postoperative approach to HCC seems to be the antiviral therapy in the case of underlying viral etiology of liver disease. A variety of drugs are included in this category, such as pegylated interferon (PEG-IFN) with or without ribavirin and nucleotide analogs regarding $\mathrm{HBV}$-related $\mathrm{HCC}$, as well as, novel direct-acting antiviral drugs for $\mathrm{HCV}$-related $\mathrm{HCC}$. The former achieves lower HBV reactivation rates and lower HCC recurrence rates at $1,2,3$ and 5 years postoperatively. The latter guarantees up to $90 \% \mathrm{HCV}$ clearance, decreased incidence of hepatic decompensation in patients who achieved sustained virologic response, as well as a significant improvement in 5-year Overall Survival (OS: 64\% vs 44\%) and increased RecurrenceFree Survival (RFS: 52\% vs 32\%). Particularly, antiviral regimens may contribute to ceasing or even improving the decline of liver function and to reducing the incidence of de novo tumors (late recurrence), while early recurrence does not seem to be affected by antiviral therapy. Consequently, both types of antiviral treatments contribute to improved clinical outcomes when it comes to $\mathrm{HBV}$ or $\mathrm{HCV}$-related $\mathrm{HCC}$ respectively and it seems to be the unique lege artis and evidence-based approach to the adjuvant setting of HCC in clinical practice. ${ }^{38,39}$

As far as other tested treatments are concerned, their clinical utility has yet to be proved and their use remains controversial. Specifically, trials regarding systematic treatment, which includes chemotherapy, sorafenib and immunotherapy, have not yielded any benefit in the recent studies. However, there are several ongoing phase III trials testing immune checkpoint blockers, in the adjuvant setting. An optimistic ongoing trial (at recruitment phase), is the 783TiP CA209-9DX, which aims to evaluate the use of adjuvant nivolumab in patients with HCC who are at high risk of recurrence after curative resection or ablation. ${ }^{40}$ Other promising, phase III trials in the same context are the KEYNOTE937, evaluating pembrolizumab, the EMERALD-2 evaluating durvalumab and the IMbrave050 testing the combination of atezolizumab plus bevacizumab. ${ }^{18}$ Moreover, the role of TACE and radiation therapy regarding adjuvant therapy of HCCs seems to be limited, whereas, contrariwise, potential benefits have been documented regarding hepatic arterial infusion (HAI) therapy and tumor ablation (RF, percutaneous ethanol injection, microwave, irreversible electroporation). ${ }^{38}$ Finally, another promising method seems to be the use of radiolabeled lipiodol, which is a safe radiotracer that leads to tumor necrosis and is associated with Disease-Free Survival (DFS) and OS benefit. ${ }^{41}$ Despite the multiple clinical trials of TKIs and ICIs, that are underway, no systematic therapy is considered standard of care in the adjuvant setting.

\section{Systemic Treatment}

Systemic treatment is recommended for advanced stage (C) HCC involving portal hypertension, extrahepatic spread and preserved liver function with Eastern Cooperative Oncology Group Performance Status Scale (ECOG PS) 1-2 by the Barcelona-Clinic Liver-Cancer (BCLC). Patients with intermediate-stage HCC, who are not candidates for TACE, are also eligible for this kind of treatment. Targeted therapies and immunotherapy have recently emerged as first-line and second- or even thirdline options. ${ }^{42}$ 


\section{First Line}

Before the introduction of VEGFR TKIs, cytotoxic agents, hormonal therapies, or combinations of these agents were tested as the main systemic chemotherapeutic options for the treatment of advanced HCC. However, these showed limited value and thus, they have never really been a standard of care in HCC. ${ }^{43}$ In 1988, a randomized controlled trial of doxorubicin showed significant survival benefit over best supportive care in patients with advanced HCC. ${ }^{44}$ However, the drug failed to be placed as a standard chemotherapy agent due to the severe adverse events it arose. ${ }^{4,45} \mathrm{Up}$ to date, four clinical trials using the chemotherapeutic schedule PIAF have been published, but none of them managed to show an increase in overall survival over doxorubicin. ${ }^{21,45}$ This was not achieved by the ALLIANCE trial either (sorafenib vs sorafenib and doxorubicin). (Table 1) ${ }^{23}$ Five randomized controlled trials with tamoxifen, octreotide, and interferon compared with best supportive care or placebo have been conducted worldwide, bearing ambivalent results and, thus, no clinical value. ${ }^{23-26}$

In the last few years, targeted multi-kinase inhibitors (TKIs) would comprise the standard of care for patients with advanced HCC. The main target of these agents is the receptor VEGFR 1-3. Besides VEGFR, each one of these agents targets a variety of other receptors, as well as signaling pathways. ${ }^{27}$ Sorafenib was the first drug approved by the FDA in 2008 for patients with HCC and, until May 2020, it was the standard of care for patients with advanced disease. ${ }^{28}$ In May 2020, the combination of atezolizumab plus bevacizumab improved OS and PFS over sorafenib in the IMbrave 150 trial $^{18}$ and became the new reference standard in the first-line HCC treatment. Except for VEGFR, sorafenib also inhibits the platelet-derived growth factor receptor (PDGFR), as well as the BRAF protein. It is recommended for patients with preserved liver function (Child-Pugh A) and advanced disease (BCLC C) or for those with an intermediatestage disease (BCLC B) and ECOG PS 0-2 that relapsed after locoregional therapies and have not received any other systematic therapy. The phase III study SHARP showed an increase in OS, from 7.9 months with placebo to 10.7 with sorafenib. ${ }^{28}$ The safety and efficacy of the drug were confirmed in the phase III Asia-Pacific trial. ${ }^{30}$ The toxicity of this treatment, which includes diarrhea, palmar-plantar erythrodysesthesia and hemorrhage had raised the question of whether the risks outweigh the benefits, however, it became established, as for a long time it remained the sole therapeutic option available to this subset of patients. ${ }^{46-48}$ Sorafenib was the only FDAapproved option for aHCC until April 2017, when regorafenib was approved for the second-line therapy, and the only approved first-line medication until lenvatinib was approved in August 2018. ${ }^{49}$

Lenvatinib, another targeted agent of the same category, was approved in August 2018, as a first-line treatment for non-excisable HCCs, adding a further four months to the median survival of patients compared to sorafenib. ${ }^{50}$ Many phase-III trials have been carried out, comparing sorafenib with other drugs, but only the REFLECT study of lenvatinib showed non-inferiority efficacy (Table 2). ${ }^{50-56}$ Specifically, lenvatinib is an inhibitor of VEGFR 1 to 3 , as well as fibroblast growth factor receptors (FGFR) 1 to 4, PDGFR alpha, KIT and RET. The most common side effects noted for both drugs were palmar-plantar erythrodysesthesia and hypertension, whereas more serious adverse effects associated with lenvatinib like renal failure, anemia, thrombocytopenia, dyspnoea and hepatic encephalopathy led to a reduction of dosage in $70 \%$ and cessation of treatment in $20 \%$ of patients. ${ }^{57}$

Finally, yet importantly, the IMbrave 150 trial in May 2020 marked the emergence of immunotherapy as a first-line treatment for non-resectable HCC. Finn et al indicated the supremacy of atezolizumab combined with bevacizumab over sorafenib in terms of OS and PFS outcomes than sorafenib. ${ }^{18}$ Specifically, 1-year OS was $67.2 \%$ (95\% CI, 61.3 to 73.1 ) with atezolizumab-bevacizumab versus $54.6 \%$ (95\% CI, 45.2 to 64.0 ) with sorafenib, whereas median PFS was estimated at 6.8 months $(95 \%$ CI, 5.7 to 8.3) versus 4.3 months ( $95 \%$ CI, 4.0 to 5.6 ) in the respective groups ( $\mathrm{HR}, 0.59 ; 95 \% \mathrm{CI}, 0.47$ to 0.76 ; $\mathrm{P}<0.001)$. Moreover, the ORR was higher $(27.3 \%)$ with atezolizumab plus bevacizumab compared with sorafenib $(11.9 \%)$. As far as the toxicity of the combination regimen is concerned, the two groups showed similar rates of grade 3 or 4 adverse events (approximately 55\% of patients who received at least one dose of each regimen). Hypertension was documented to be the major grade 3 or 4 side effect of the combination regimen, which occurred in $15.2 \%$ of patients who received atezolizumab plus bevacizumab. Of note, patients with a history of co-infection with hepatitis $\mathrm{B}$ and hepatitis $\mathrm{C}$ virus, incompletely treated esophageal or gastric varices, high risk of bleeding or autoimmune diseases, such as autoimmune hepatitis, were excluded from this trial. ${ }^{18,58}$ The current NCCN 
Table I The History of Agents Used in the Treatment of HCC

\begin{tabular}{|c|c|c|c|}
\hline Name of the Agent & Trial & Date & Hazard Ratio $(95 \% \mathrm{Cl})$ and OS (Months) \\
\hline \multicolumn{4}{|c|}{ Ist Line } \\
\hline $\begin{array}{l}\text { Doxorubicin vs No } \\
\text { therapy }\end{array}$ & $\begin{array}{l}\text { Doxorubicin vs no antitumor therapy in aHCC. } \\
\text { A prospective randomized trial }\end{array}$ & 1988 & 10.6 weeks vs 7.5 weeks $(p=0.036)^{44}$ \\
\hline Sorafenib vs Placebo & SHARP trial (phase III) & 2008 & $0.69(0.55-0.87) ; 10.7$ vs $7.9^{28}$ \\
\hline Sorafenib vs Placebo & Asia-Pacific trial (phase III) & 2009 & $0.68(0.50-0.93) ; 6.5$ vs $4.2^{30}$ \\
\hline Sunitinib vs Sorafenib & SUNII70 (phasellI) & 2013 & $1.30(1.13-1.50) ; 7.9$ vs $10.2^{51}$ \\
\hline Brivanib vs Sorafenib & BRISK-FL (phase III) & 2013 & 1.06 (0.93-I.22); 9.5 vs $9.9^{52}$ \\
\hline Linifanib vs Sorafenib & LIGHT (phase III) & 2014 & $1.046(0.896-1.221) ; 9.1$ vs $9.8^{53}$ \\
\hline $\begin{array}{l}\text { Sorafenib }+ \text { Erlotinib vs } \\
\text { Sorafenib alone }\end{array}$ & SEARCH (phase III) & 2015 & $0.929(0.78 \mathrm{I}-\mathrm{I} .106) ; 9.5$ vs $8.5^{56}$ \\
\hline Y40 vs Sorafenib & SARAH (phase III) & 2017 & I.I5 (0.94-I.4I); 8.0 vs $9.9^{54}$ \\
\hline Y40 vs Sorafenib & SIRveNIB (phase III) & 2018 & I.I (0.9-I.4); 8.8 vs $10.0^{55}$ \\
\hline $\begin{array}{l}\text { Lenvatinib vs Sorafenib } \\
\text { (non- inferiority) }\end{array}$ & REFLECT (phase III) & 2018 & $0.92(0.79-1.06) ; 13.6$ vs $12.3^{50}$ \\
\hline $\begin{array}{l}\text { Sorafenib + Doxorubicin } \\
\text { vs Sorafenib alone }\end{array}$ & ALLIANCE (CALGB 80802) (phase III) & 2019 & 1.05 (0.83-I.3I); 9.3 vs $9.4^{23}$ \\
\hline Nivolumab vs Sorafenib & CheckMate 459 (phase III) & 2019 & 0.85 (0.72-1.02); 16.4 vs $14.7^{70}$ \\
\hline $\begin{array}{l}\text { Atezolizumab plus } \\
\text { Bevacizumab vs Sorafenib }\end{array}$ & IMbraveI50 (phase III) & 2020 & $\begin{array}{c}0.58 \text { (0.42-0.79); } 6 \text {-mo OS: } 84.8 \% \text { vs } 67.2 \% \\
\text { I2-mo OS: } 72.2 \% \text { vs } 54.6 \%^{18}\end{array}$ \\
\hline \multicolumn{4}{|c|}{ 2nd Line } \\
\hline Regorafenib & RESORCE (phase III) & 2016 & $0.63(0.50-0.79) ; 10.6$ vs $7.8^{16}$ \\
\hline Nivolumab & CheckMate 040 (phase I/II) & 2017 & OS $(95 \% \mathrm{Cl}): 15.1(13.2-18.2)^{67}$ \\
\hline Cabozantinib & CELESTIAL (phase III) & 2018 & $0.76(0.63-0.92) ; 10.2$ vs $8.0^{60}$ \\
\hline Ramucirumab & REACH (phase III) & 2015 & $0.87(0.72-1.05) 9.2$ vs $7.6^{63}$ \\
\hline Ramucirumab & REACH-2 (phase III) & 2018 & $0.71(0.53-0.95) 8.5$ vs $7.3^{64}$ \\
\hline Pembrolizumab & KEYNOTE-224 (phase II) & 2018 & $\begin{array}{c}\text { OS (95\% Cl): I } 3.2(9.7-15.5), \text { I-year OS (95\% Cl): } 54 \% \\
(44-63)^{68}\end{array}$ \\
\hline Pembrolizumab & KEYNOTE-240 (phase III) & 2020 & $0.78(0.61-1.00) 13.9$ vs $10.6^{69}$ \\
\hline $\begin{array}{l}\text { Nivolumab plus } \\
\text { Ipilimumab }\end{array}$ & CheckMate 040 (phase II) & 2020 & $\begin{array}{l}22.8 \text { (9.4-not reached), I-year OS (95\% Cl): 61\% } \\
(46-73 \%), 2 \text {-year OS (95\% Cl): } 48 \%(34-61 \%)^{80}\end{array}$ \\
\hline
\end{tabular}

Abbreviations: aHCC, advanced hepatocellular carcinoma; vs, versus; Y-40, Yttrium-40; OS, overall survival.

guideline recommends sorafenib or lenvatinib in the firstline treatment of aHCC patients who are not appropriate for atezolizumab plus bevacizumab. ${ }^{50}$ Nonetheless, combining immunotherapy with VEGF inhibitors in the firstline treatment of advanced hepatocellular carcinoma is expected to be the next step in the management of HCC. ${ }^{58}$

\section{Second and Third Line}

It took nine years until 2017 when another agent called regorafenib was added in the armamentarium of HCC treatments. The phase-III trial, RESORCE, compared regorafenib with placebo and showed an advantage in survival (from 7.8 to 10.6 months). ${ }^{16}$ Regorafenib is 
Table 2 Approved Systematic Therapies for HCC

\begin{tabular}{|l|l|c|c|}
\hline I st Line Treatment & Targets & EMA & FDA \\
\hline Sorafenib & VEGFR I-3, PDGFR- $\beta$, KIT kinases & V \\
\hline Lenvatinib & VEGFR I-3, FGFR I-4, PDGFR $\alpha$, RET, KIT & V \\
\hline Atezolizumab + Bevacizumab & PD-LI + VEGF-a & V & V \\
\hline 2nd LINE TREATMENT & & V \\
\hline Regorafenib & VEGFR, PDGFR, BRAF, KIT, RET, RAF-I, FGFR, Tie2 & V \\
\hline Cabozantinib & VEGFR 2, aMET, AXL, RET, c-MET & V \\
\hline Ramucirumab & VEGFR 2 & V \\
\hline Nivolumab & PD-I & V \\
\hline Pembrolizumab & PD-I & V \\
\hline Nivolumab + Ipilimumab & PD-I + CTLA-4 & $X$ \\
\hline
\end{tabular}

Abbreviations: EMA, European Medicines Agency; FDA, food and drug administration; VEGFR, vascular endothelial growth factor receptor; PDGFR- $\beta$, platelet-derived growth factor beta; FGFR, fibroblast growth factor receptors; PD-LI, programmed death-ligand I; VEGF-a, vascular endothelial growth factor alpha; Tie2, a tyrosine kinase receptor; c-MET, tyrosine-protein kinase met or hepatocyte growth factor receptor (HGFR); PD-I, programmed cell death protein I; CTLA-4, cytotoxic T-lymphocyteassociated protein 4 ; $V$, yes; $X$, no.

a tyrosine kinase inhibitor with anti-angiogenic properties, similar to sorafenib. It was first introduced as a second-line treatment combined with sorafenib, where it added an extra 2.8 months to the overall survival of patients. It has been approved for patients with intermediate or advanced stage HCC (BCLC B or C), Child-Pugh A, ECOG PS 0-1, and those who have relapsed after treatment with sorafenib. $^{49}$ Regorafenib improved OS with a hazard ratio of 0.63 . Its median survival was 10.6 months versus 7.8 months for placebo. The median time to progression was 3.2 months with regorafenib and 1.5 months with placebo. One percent of the patients in the regorafenib group versus no patients in the placebo group had a complete response. ${ }^{16,22}$ Hypertension, hand-foot syndrome, fatigue and diarrhea are some striking examples of the major side effects of this agent. ${ }^{59}$

At the second-line treatment status, cabozantinib, a TKI targeting the VEGFR2, aMET, AXL and RET inhibitors, has been demonstrated as an alternative to regorafenib. The CELESTIAL phase-III trial showed an increase in survival from 8 to 10.2 months. PFS was increased to 5.2 months with cabozantinib. The disease progression rate was 0.36 with cabozantinib and 0.52 with placebo; $(\mathrm{P}<0.001)$, and the ORRs were $4 \%$ and less than $1 \%$, respectively. ${ }^{60}$ In contrary to RESORCE, the CELESTIAL trial included patients with intolerance to regorafenib, as well as progressive disease after one or two systemic treatments. ${ }^{61}$ Hand-foot syndrome, hypertension, aspartate transaminase (AST) increase, weakness and diarrhea were amongst the most common side effects. ${ }^{62}$ Based on this trial, cabozantinib gained FDA approval in January 2019 in the second-line setting.

Furthermore, ramucirumab, a human monoclonal antiVEGFR 2 antibody, is included in this category of agents. The phase-III REACH trial showed some slight increase in survival in a subgroup of patients with AFP $>400 \mathrm{ng} / \mathrm{mL}$ when they received ramucirumab as a second-line of treatment following sorafenib, due to either progression of the disease or intolerance to sorafenib. ${ }^{63}$ Based on this observation, the REACH-2 trial showed an increase in survival from 7.3 to 8.5 months in this subgroup of patients, compared to placebo. PFS was increased to 2.8 months from 1.6 months, while no difference was documented in terms of OR. ${ }^{64}$ Some common side effects which were documented were hypertension and hyponatremia. $^{65}$ Therefore, ramucirumab $(8 \mathrm{mg} / \mathrm{kg}$ once every 2 weeks) was approved by the FDA in May 2019 for patients with aHCC and AFP of $\geq 400 \mathrm{ng} / \mathrm{mL}$ in the secondline setting. In a meta-analysis of HCC studies including $\mathrm{REACH}$ and $\mathrm{REACH}-2$, ramucirumab provided survival benefit in patients who did or did not experience ascites in the second-line treatment of HCC. The OS was 6.7 vs 3.4 months in ramucirumab and placebo arms with HR of 0.3 $(0.18,0.49)$, and the PFS was 4.2 vs 2 months with HR 0.46 $(0.29,0.74)$ in patients with aHCC who experience ascites. ${ }^{66}$ 
Checkpoint inhibitors such as nivolumab and pembrolizumab, which have both completed Phase II trials with encouraging outcomes, are also recommended as secondline treatments given that the aforementioned antiangiogenic agents are associated with significant toxicity compared to immunotherapy. ${ }^{67,68}$ Recently, the phase I/II trial CheckMate 040 of nivolumab, which included patients who had already received sorafenib, revealed an overall survival of 15.6 months. The ORR was $20 \%$ during the dose-expansion phase with patients treated with nivolumab $\left(3 \mathrm{mg} / \mathrm{kg}\right.$ ) and $15 \%$ in the dose-escalation phase ${ }^{69}$ However, so far phase III trials have failed to deliver statistically significant results. ${ }^{69,70}$ Moreover, it is worth mentioning that response rates of these immune checkpoint inhibitors do not exceed $25 \%$, whereas grade $3 / 4$ treatment of immunerelated adverse events, such as rash, diarrhea, pruritus and increase of transaminases, have been documented. ${ }^{19}$ The FDA approved nivolumab (240mg every 2 weeks) in the second-line setting in September $2017 . .^{71}$ It is also recommended in the front-line setting if patients are considered ineligible or intolerant to other front-line medications. Pembrolizumab, on the other hand, being assessed in the single-arm Keynote-224 trial gained FDA approval in the second-line setting for patients with aHCC in November 2018. Recently approved, also in the secondline setting for the treatment of aHCC, was the combination of nivolumab plus ipilimumab. ${ }^{72,73}$

\section{Implications for Clinical Practice}

Currently, according to the presented evidence, it seems that HCC management can be vaguely divided between the management of resectable and non - resectable tumors. In the first case, surgery can be preceded or followed by various therapeutic modalities including TACE and TARE (sorafenib and ICIs are also being assessed in phase II trials) as neoadjuvant interventions ${ }^{32-35}$ or systematic therapy, hepatic arterial infusion therapy, TACE, radiolabeled lipiodol, ablation and radiotherapy postoperatively. ${ }^{37-39}$ As far as non-resectable tumors are concerned, TKIs and immunotherapy have now replaced chemotherapy and hormone therapy. Particularly, the novel doublet of atezolizumab plus bevacizumab is indicated as a first-line option for patients (Child-Pugh A, ECOG PS 0-1) without a history of co-infection with hepatitis $\mathrm{B}$ and hepatitis $\mathrm{C}$, autoimmune disease, or bleeding predisposition. ${ }^{18}$ Lenvatinib is considered a first-line treatment for non-excisable HCCs, while sorafenib is recommended for patients with preserved liver function (Child-Pugh A) and advanced disease
(BCLC C) or those with an intermediate-stage disease (BCLC B) and ECOG PS 0-2 relapsing after locoregional therapies and having received no other systematic therapy. ${ }^{50}$ Sorafenib or lenvatinib in the first-line setting are recommended by the NCCN guidelines for the treatment of aHCC patients who are not appropriate for atezolizumab plus bevacizumab. ${ }^{28,50}$

When it comes to second-line treatment, regorafenib and cabozantinib have been approved for patients with intermediate or advanced stage HCC (BCLC B or C), Child-Pugh A, ECOG PS $0-1$, and those who have relapsed after treatment with sorafenib. ${ }^{22,63}$ Similarly, ramucirumab is recommended as a second-line treatment following sorafenib, due to either progression of the disease or intolerance to sorafenib. ${ }^{63,64}$ Although nivolumab, pembrolizumab or ramucirumab remain second-line choices after failure of atezolizumab plus bevacizumab according to the updated ESMO guidelines, further investigation is needed for them to be established. Finally, the combination of nivolumab and ipilimumab has already been incorporated in the armamentarium of second-line treatments for HCC as meeting the endpoints of the combination arm of the CheckMate-040 study and it was approved by the FDA in March $2020^{71}$ (Figure 1).

\section{The Current Issues}

These advances can be discussed in the context of HCC, but they should also be addressed in the bigger picture. As far as the treatment of HCC is directly concerned, despite the progress in targeted therapies, immunotherapy still plays a minor role in HCC's treatment. The role of immunotherapy, and particularly PD-1/PD-L1 ought to be

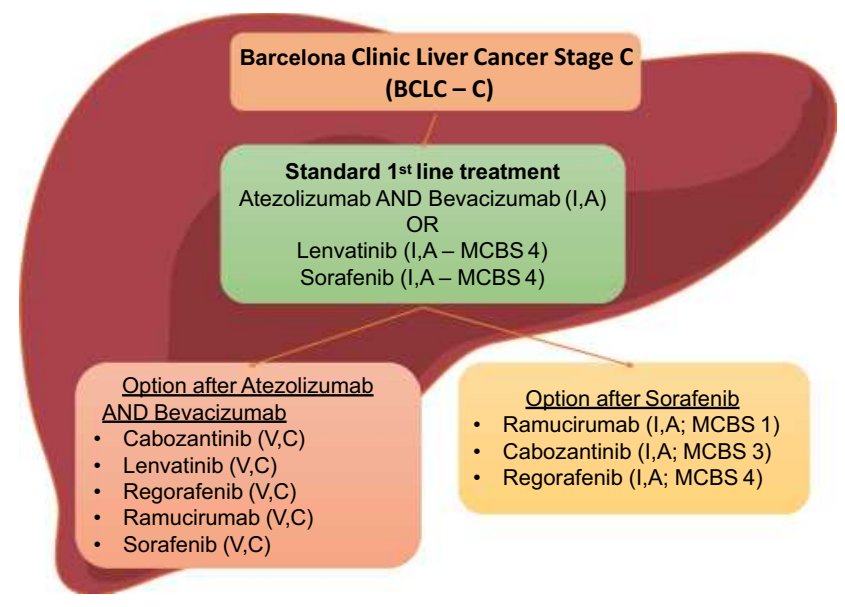

Figure I Treatment algorithm for $\mathrm{HCC}{ }^{42,66}$

Abbreviation: MCBS, Magnitude of Clinical Benefit Scale. 
reassessed and upgraded. Recent studies have shown an increasing interest to this end. In any case, the limited efficacy of conventional anticancer therapies such as chemotherapy and hormonal therapy has opened the door for biologics, setting an example for other types of cancer, where conventional means have repeatedly failed. ${ }^{68-71}$

TKIs, such as sorafenib and lenvatinib recommended as a first-line treatment regarding HCC, followed by a secondline treatment with a different TKI, such as regorafenib. ${ }^{74}$ Moreover, the two novel anti-angiogenic factors cabozantinib and ramucirumab have successfully completed phase III trials and have, consequently, been added as a second-line treatment option. ${ }^{75}$ Specifically, ramucirumab can be used in the subset of patients with AFP $>400 \mathrm{ng} / \mathrm{mL}$ and intolerance to TKIs. ${ }^{76}$ The toxicity of these drugs, in addition to their lower objective response rates, compared to immunotherapy, are fields seeking improvement. ${ }^{19}$ Combinations of the aforementioned targeted therapies with immunotherapy are tested as first-line treatment in current clinical trials. ${ }^{58,77}$ The use of immunotherapy with PD-L1 inhibitors as single agents or in combination with another immune checkpoint inhibitor, with TKIs or with other anti-angiogenic agents might lead to the redefinition, not only of the therapeutic algorithm but of the whole therapeutic strategy. Finally, because of the plethora of novel therapeutic choices, the correct course of later lines of treatment is open to debate and has to be validated through further research (Figure 2).

Until recently, immunotherapy was limited to a subset of patients with ECOG PS $0-1$ and progressive disease after TKI therapy. ${ }^{78}$ It is worth noting that phase III trials have yet to be completed for immunotherapy agents, which are expected to further modify the list of treatments available for the malignancy in question. Many phase III trials had failed to prove superiority and place these agents amongst the first-line treatment options. Particularly, the phase III CheckMate 459 trial, which compared nivolumab to sorafenib as a first-line treatment, failed to show statistical significance. $^{70}$ The results of the phase II KEYNOTE-224 trial of another anti-PD-1 monoclonal antibody, named pembrolizumab, as a second-line treatment, were released at the 2019 ASCO Meeting. This study failed to reach the PFS and OS targets that were set, despite increasing the PFS and the

\section{Sorafenib vs placebo \\ CTP, ECOG PS $\leq 2$}

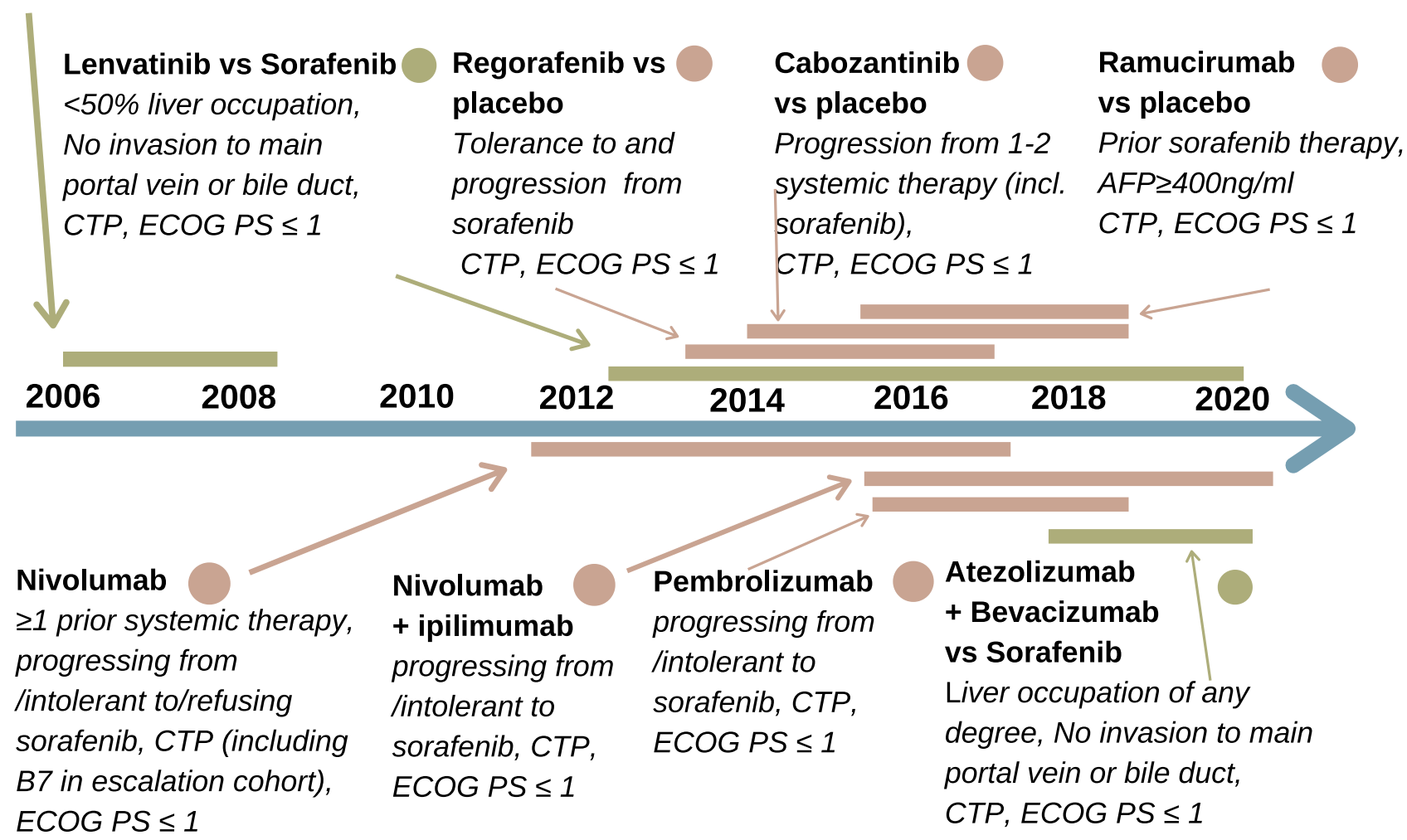

Figure 2 HCC treatment timeline. ${ }^{11,12,17,18}$

Abbreviations: ECOG PS, Eastern Cooperative Oncology Group Performance Status; CTP, Child-Turcotte-Pugh classification. 
OS to 4.9 months. ${ }^{16}$ On the contrary of all these, IMbrave150 managed to prove benefit and establish the atezolizumab plus bevacizumab combination as the new reference standard in the first-line. New horizons are now open for investigation and research in the immunotherapy setting (Figure 3).

As already mentioned, not only has immunotherapy been tested as monotherapy, but it has also been incorporated in combination regimens in a plethora of ongoing trials. $^{79-90}$ A striking example is the combined treatment of nivolumab plus ipilimumab, which led to significant objective response rates (ORR: $32 \%$ ) and durable responses, but it also revealed increased toxicity compared to monotherapy with nivolumab (ORR: 14\%). However, the combination regimen (nivolumab $1 \mathrm{mg} / \mathrm{kg}$ plus ipilimumab $3 \mathrm{mg}$ / kg every 3 weeks for 4 cycles and then nivolumab $240 \mathrm{mg}$ every 2 weeks) received accelerated approval in the USbased on the phase III CheckMate-040 clinical trial. ${ }^{71,80}$
Recently, the aforementioned IMbrave 150 study, which evaluates the combination of atezolizumab plus bevacizumab in the first-line treatment of advanced HCC, was published. It was the first positive phase III trial regarding combination therapy with an immune checkpoint inhibitor (PDL-1 inhibitor) and a targeted agent (VEGF inhibitor) compared to sorafenib, which has shown OS improvement since $2008 .^{58}$ This practice-changing study may evangelize a new approach in the management of HCC, which will be based on the combinations between immunotherapy and anti-angiogenic therapy.

From a pathophysiologic perspective, simultaneous targeting of tumor vessels and immunity is a promising strategy to normalize aberrant vascular-immune crosstalk and potentiate immunotherapy. This may entail a favorable regulation of the tumor microenvironment (TME). Particularly, the malformed tumor vessels prevent

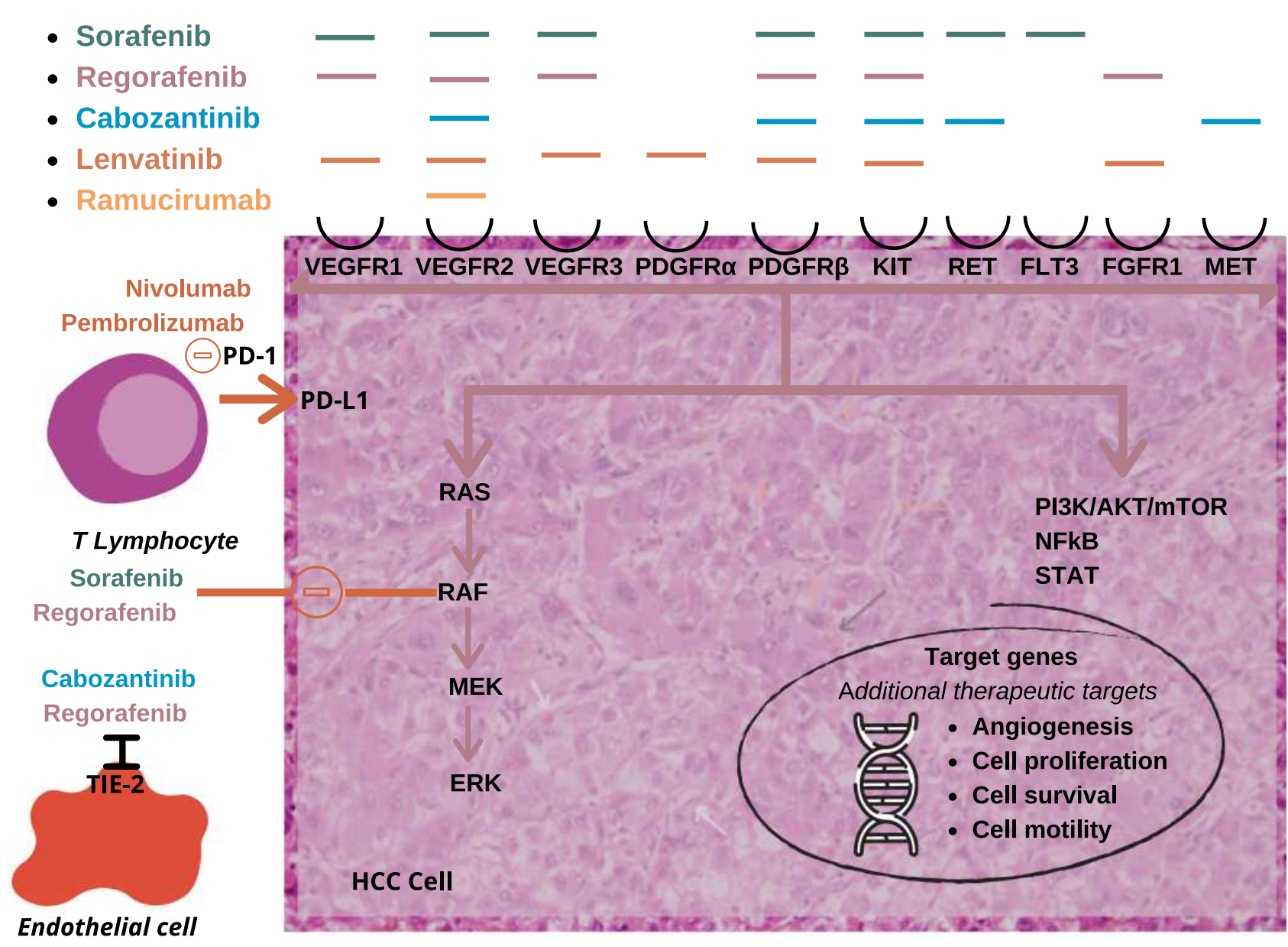

Figure 3 Treatment regimens and their mechanisms of action. The Ras/Raf/MEK/ERK and PI3K/Akt/mTOR cascades are often activated by genetic alterations in upstream signaling molecules such as receptor tyrosine kinases. ${ }^{27,77,78}$

Abbreviations: HCC, hepatocellular carcinoma; VEGFR, vascular endothelial growth factorrReceptor 2; PDGFR, platelet-derived growth factor; FLT3, fms-like tyrosine kinase 3; FGFR, fibroblast growth factor receptors; MET, tyrosine-protein kinase met or hepatocyte growth factor receptor (HGFR); Tie2, a tyrosine kinase receptor; PD-I, programmed cell death protein I; PD-LI, programmed death-ligand I. 
$\mathrm{CD} 8+\mathrm{T}$ and $\mathrm{CD} 4+\mathrm{TH} 1$ cells from transmigration into the TME, disable their effector functions and even lead to their apoptosis. Furthermore, VEGF is involved in the growth of dendritic cells, thus, suppressing $\mathrm{T}$ cell priming, and, finally, stimulating TOX-mediated exhaustion of CD8 $+\mathrm{T}$ cells. On the other hand, the malformation of tumor vessels is enabled by a plethora of innate and adaptive immune cells, such as pro-tumoral M2-like macrophages, TH2 and Treg cells. These cells secrete pro-angiogenic factors that promote unrestrained angiogenesis and contribute to vascular immaturity. Preclinical data as those mentioned above have already been translated into clinical benefit and have shown the way to the future. $^{81}$

\section{Future Perspectives}

Testing a variety of agents and combinations in patients with HCC is of paramount importance. Therefore, at present, numerous clinical trials are underway. ${ }^{82-84}$ Remarkably, a Phase I trial of the combination of pembrolizumab and lenvatinib, which was well tolerated with promising anti-tumor activity in patients with unresected HCC. ${ }^{85,90}$ Furthermore, it is worth mentioning that there are two ongoing phase III studies, the COSMIC-312 study and the HIMALAYA study, in which combination regimens are being evaluated. The former study refers to the combination of cabozantinib plus atezolizumab versus sorafenib in patients with advanced HCC who receive their first-line of treatment and the latter regards the combination of an anti-CTLA-4 monoclonal antibody (Cytotoxic $\mathrm{T}$ lymphocyte-associated antigen), named tremelimumab, with an anti-PDL-1 immune agent, named durvalumab. ${ }^{86-88}$ The HIMALYA trial revealed tolerable toxicity and encouraging activity in patients with advanced HCC. ${ }^{87}$ Moreover, monotherapy with avelumab, an antiPDL1 antibody, and single-agent ipilimumab is currently being tested. Notably, it is alarming that the majority of the ongoing clinical trials are comparing the potential treatment choices with sorafenib instead for the new goldstandard of bevacizumab plus atezolizumab as the firstline treatment of advanced HCC. Finally, the experimental investigation of novel sophisticated treatments, such as adoptive cell therapy using T-cell engineering, cancer vaccines and oncolytic virus therapies form an evolving field, which promotes an unprecedented approach to the systematic management of advanced HCC (Table 3). ${ }^{89}$

As far as biomarkers are concerned, there are a plethora of intra-tumoral and extra-tumoral biomarkers, which are currently evaluated with the object of bridging the aforementioned biomarkers gap. Particularly, research seems to place primacy on biomarkers of response to immunotherapy. Beyond PDL-1 expression, which is the most widely used immune biomarker in clinical practice: Tumor Mutational Burden (TMB), the density of Tumor-Infiltrating Lymphocytes (TILS), peripheral immune cells' phenotype, cell-free DNA (cfDNA) and gut microbiome/ microbiota are included in the potential and emerging predictive biomarkers regarding multiple cancer types. ${ }^{91}$ Nevertheless, when it comes to HCC, the intra-tumoral PDL-1 expression was extremely low and heterogeneous with a subsequent dramatic effect on its predictive value, according to CheckMate 040 and Keynote 224 trials. $^{70,71}$ On the contrary, increased infiltration of CD8+ T-cell, effector T-cells, or cytolytic T-cell into the tumor was related to response to immunotherapy in HCC. What is more, lower tumor transcriptomic diversity was associated with PFS and OS of patients with HCC who were treated with a combination of immune agents, whereas WNT pathway mutations were associated with resistance to immunotherapy ${ }^{89,92}$ As far as extra-tumoral biomarkers are concerned, circulating tumor DNA could be distinguished, given that it has been documented that cfDNA with high mutational burden is related to clinical outcome in patients who received immunotherapy. ${ }^{93}$ Additionally, it is worth mentioning that an immune gene expression signature, which is called immune-mediated cancer field (ICF) is evaluated in liver tissues of patients with early-stage HCC so that the risk of HCC development in patients with cirrhosis can be assessed. It has been demonstrated that anti-inflammatory agents, such as aspirin and nintedanib cause loss of this gene expression in mice which developed less aggressive malignancies with smaller and fewer liver tumors. ${ }^{94}$

Another promising ally of the oncological community against cancer may be found in the gradually increasing use of biomaterials in medicine. Specifically, biomaterials include a wide range of nanoparticles to macroscopic compounds, which can facilitate both local and systematic delivery of HCC drugs. Consequently, besides the bioavailability benefit of biomaterials, the interest of some HCC drugs that failed to prove any efficacy via systematic delivery, may then be reignited. ${ }^{95}$ Moreover, biomaterials may be applicable for diagnostic reasons through specific electrospun nanofibers, which result in capturing CTCs. ${ }^{96}$ 
Table 3 Indicative List of Ongoing Clinical Trials

\begin{tabular}{|c|c|c|}
\hline Regimen & Outcome & Study Name \\
\hline Pembrolizumab plus Lenvatinib & $\begin{array}{l}\text { Well-tolerated and encouraging anti-tumor activity in aHCC (mPFS: } \\
\qquad 9.3 \text { months per mRECIST) }\end{array}$ & $\left(\right.$ phase lb) ${ }^{85,90}$ \\
\hline Tremelimumab plus Durvalumab & Well-tolerated and promising activity in aHCC and BTC & HIMALAYA (phase III) ${ }^{87,88}$ \\
\hline $\begin{array}{l}\text { Nivolumab plus Ipilimumab and } \\
\text { Nivolumab plus Ipilimumab plus } \\
\text { Cabozantinib }\end{array}$ & $\begin{array}{l}\text { Clinically meaningful responses versus nivolumab monotherapy and } \\
\text { acceptable safety profile in sorafenib treated pts, (mPFS: } 5.5 \text { months } \\
\text { for the doublet regimen and } 6.8 \text { months for the triplet regimen, OS } \\
\text { not reached in either arm) }\end{array}$ & CheckMate-040 (phase I/II) \\
\hline $\begin{array}{l}\text { GNOS-PV02 plus Vaccine INO- } \\
9012 \text { plus Pembrolizumab }\end{array}$ & $\begin{array}{c}\text { Evaluation of a Personalized Neoantigen DNA Vaccine (GNOS-PV02) } \\
\text { and Plasmid Encoded IL-I2 (INO-90I2) in combination with } \\
\text { Pembrolizumab in aHCC }\end{array}$ & Phase $\mathrm{I} / \mathrm{Il}{ }^{83}$ \\
\hline Nivolumab plus Ipilimumab & $\begin{array}{l}\text { Compared to sorafenib or lenvatinib as first-line treatment in } \\
\text { participants with aHCC }\end{array}$ & CheckMate-9DW (phase III) ${ }^{82}$ \\
\hline Cabozantinib + Atezolizumab & $\begin{array}{l}\text { Compared to Sorafenib as first line treatment. Primary endpoints: PFS } \\
\text { and OS. }\end{array}$ & COSMIC-3I2 (phase III) ${ }^{82,84,86}$ \\
\hline Lenvatinib + Pembrolizumab & Promising antitumor activity and acceptable safety profile in aHCC. & LEAP-002 (phase III) ${ }^{82,84}$ \\
\hline $\begin{array}{l}\text { Sorafenib }+ \text { pexastimogene } \\
\text { devacirepvac (Pexa-Vec) }\end{array}$ & $\begin{array}{l}\text { Acceptable safety profile and increased OS. Response by modified } \\
\text { RECIST criteria: I5\% and Response by Choi: } 62 \% \text {. Intrahepatic DCR: } \\
50 \% \text {. Median OS: } 14.1 \text { months with the high dose versus } 6.7 \text { months } \\
\text { with the low dose (HR, } 0.39 ; P=0.02)\end{array}$ & PHOCUS (phase III) ${ }^{84}$ \\
\hline Atezolizumab + Bevacizumab & HR: $0.66(95 \% \mathrm{Cl}, 0.52-0.85) ; 19.2$ vs 13.4 & IMbrave I50 (phase III) ${ }^{18}$ \\
\hline BGB-A3I7 (Tislelizumab) & $\begin{array}{l}\text { Well-tolerated and promising antitumor activity in patients with } \\
\text { advanced HCC in early phases trials. } \\
\text { A Phase III, randomized, open-label study is active (compared to } \\
\text { sorafenib as a first-line treatment in aHCC). }\end{array}$ & RATIONALE $30 \mathrm{I}$ (phase III) ${ }^{82,84}$ \\
\hline
\end{tabular}

Abbreviations: aHCC, advanced hepatocellular carcinoma; mPFS, median progression-free survival; BTC, biliary tract carcinoma; OS, overall survival; DCR, disease control rate; HR, hazard ratio.

Further investigation about their sensitivity and specificity rates is needed. Last but not least, given the ability of HCC-derived organoids to illustrate the genetic complexity of the tumor, they consist potential tools for personalized biomarker identification and drug screening. Thus, biomaterial-based applications may soon render them as one of the foremost exponents of personalized medicine. More specifically, tissue engineering 3D models may be beneficial for achieving a deeper understanding of cancer biology and for developing effective therapies for HCC, following the principles of precision medicine. $^{97}$

\section{Conclusion}

In conclusion, rapidly emerging new data have drastically changed the landscape when it comes to HCC, which is now well defined as an oncological entity.
Diagnosis can now be achieved using non-invasive imaging techniques and there are a plethora of treatment options available. Correct use of newly established treatments, however, remains a challenge, as the current holistic approach to cancer, raises the need for "tailored" treatments, that contribute not only to the overall survival of patients but to their quality of life as well. Finally, these emerging developments introduce the "medical community" to a new era regarding the management of HCC. An era that entails a multidisciplinary approach, based on genuine cooperation between medical specialties.

\section{Disclosure}

The authors declare that there is no conflicts of interest. 


\section{References}

1. Akinyemiju T, Akinyemiju T, Abera S; Global Burden of Disease Liver Cancer Collaboration, et al. The burden of primary liver cancer and underlying etiologies from 1990 to 2015 at the global, regional, and national level: results from the global burden of disease study 2015 . JAMA Oncol. 3;2017:1683-1691. doi:10.1001/jamaoncol.2017.3055

2. Ferlay J, Soerjomataram I, Dikshit R, et al. Cancer incidence and mortality worldwide: sources, methods and major patterns in GLOBOCAN 2012. Int $J$ Cancer. 2015;136:E359-E386. doi:10.1002/ijc.29210

3. Ryerson AB, Eheman CR, Altekruse SF, et al. Annual report to the nation on the status of cancer, 1975-2012, featuring the increasing incidence of liver cancer. Cancer. 2016;122:1312. doi:10.1002/cncr.29936

4. Quaglia A. Hepatocellular carcinoma: a review of diagnostic challenges for the pathologist. J Hepatocell Carcinoma. 2018;5:99. doi:10.2147/JHC.S159808

5. Pennisi G, Celsa C, Giammanco A, et al. The burden of hepatocellular carcinoma in non-alcoholic fatty liver disease: screening issue and future perspectives. Int $J$ Mol Sci. 2019;20(22):5613. doi:10.3390/ijms20225613

6. Mittal S, El-Serag HB, Sada YH, et al. Hepatocellular carcinoma in the absence of cirrhosis in United States veterans is associated with nonalcoholic fatty liver disease. Clin Gastroenterol Hepatol. 2016;14:124. doi:10.1016/j.cgh.2015.07.019

7. Maucort-Boulch D, de Martel C, Franceschi S, et al. Fraction and incidence of liver cancer attributable to hepatitis $\mathrm{B}$ and $\mathrm{C}$ viruses worldwide. Int J Cancer. 2018;142:2471. doi:10.1002/ijc.31280

8. Ascha MS, Hanouneh IA, Lopez R, et al. The incidence and risk factors of hepatocellular carcinoma in patients with nonalcoholic steatohepatitis. Hepatology. 2010;51:1972. doi:10.1002/hep.23527

9. Maan R, Feld JJ. Risk for hepatocellular carcinoma after hepatitis c virus antiviral therapy with direct-acting antivirals: case closed? Gastroenterology. 2017;153:890. doi:10.1053/j.gastro.2017.08.052

10. Forner A, Reig M, Bruix J. Hepatocellular carcinoma. Lancet. 2018;391:1301

11. European Association for the Study of the Liver. European Association for the Study of the Liver. EASL clinical practice guidelines: management of hepatocellular carcinoma. $J$ Hepatol. 2018;69:182

12. Marrero JA, Kulik LM, Sirlin CB, et al. Diagnosis, staging, and management of hepatocellular carcinoma: 2018 practice guidance by the American Association for the Study of Liver Diseases. Hepatology. 2018;68:723. doi:10.1002/hep.29913

13. Nault JC, Sutter O, Nahon P, et al. Percutaneous treatment of hepatocellular carcinoma: state of the art and innovations. $J$ Hepatol. 2018;68:783. doi:10.1016/j.jhep.2017.10.004

14. Palmer DH. Radiofrequency ablation with or without transcatheter arterial chemoembolization. $J$ Clin Oncol. 2013;31:2756. doi:10.1200/JCO.2013.49.8352

15. Jackson R, Psarelli -E-E, Berhane S, et al. Impact of viral status on survival in patients receiving sorafenib for advanced hepatocellular cancer: a meta-analysis of randomized phase III trials. J Clin Oncol. 2017;35:622. doi:10.1200/JCO.2016.69.5197

16. RESORCE Investigators; Bruix J, Qin S, Merle P, et al. Regorafenib for patients with hepatocellular carcinoma who progressed on sorafenib treatment (RESORCE): a randomised, double-blind, placebo-controlled, Phase 3 trial. Lancet. 2017;389(10064):56-66. doi:10.1016/S0140-6736(16)32453-9.

17. Bouattour M, Mehta N, He A, et al. Systemic treatment for advanced hepatocellular carcinoma. Liver Cancer. 2019;8(5):341-358. doi:10.1159/000496439

18. IMbrave150 Investigators; Finn RS, Qin S, Ikeda M, et al. Atezolizumab plus bevacizumab in unresectable hepatocellular carcinoma. N Engl J Med. 2020;382(20):1894-1905. doi:10.1056/ NEJMoa1915745.
19. Lleo A, Rimassa L, Colombo M, et al. Hepatotoxicity of immune check point inhibitors: approach and management. Dig Liver Dis. 2019;51(8):1074-1078. doi:10.1016/j.dld.2019.06.017

20. Doycheva I, Thuluvath PJ. Systemic therapy for advanced hepatocellular carcinoma: an update of a rapidly evolving field. J Clin Exp Hepatol. 2019;9(5):588-596. doi:10.1016/j.jceh.2019.07.012

21. Qin S, Bai Y, Lim HY, et al. Randomized, multicenter, open-label study of oxaliplatin plus fluorouracil/leucovorin versus doxorubicin as palliative chemotherapy in patients with advanced hepatocellular carcinoma from Asia. J Clin Oncol. 2013;31:3501. doi:10.1200/ JCO.2012.44.5643

22. Thillai K, Srikandarajah K, Ross P, et al. Regorafenib as treatment for patients with advanced hepatocellular cancer. Future Oncol. 2017;13 (25):2223-2232. doi:10.2217/fon-2017-0204

23. Abou-Alfa GK, Shi Q, Knox JJ, et al. Assessment of treatment with sorafenib plus doxorubicin vs sorafenib alone in patients with advanced hepatocellular carcinoma: phase 3 CALGB 80802 randomized clinical trial. JAMA Oncol. 2019;5:1582. doi:10.1001/jamaoncol.2019.2792

24. Treiber G. Systemic treatment of hepatocellular carcinoma. Dig Dis. 2001;19(4):311-323.

25. Verset G, Verslype C, Reynaert H, et al. Efficacy of the combination of long-acting release octreotide and tamoxifen in patients with advanced hepatocellular carcinoma: a randomised multicentre phase III study. Br J Cancer. 2007;97(5):582-588.

26. Shaaban S, Negm A, Ibrahim EE, et al. Chemotherapeutic agents for the treatment of hepatocellular carcinoma: efficacy and mode of action. Oncol Rev. 2014;8(1):246.

27. Liu L, Cao Y, Chen C, et al. Sorafenib blocks the RAF/MEK/ERK pathway, inhibits tumor angiogenesis, and induces tumor cell apoptosis in hepatocellular carcinoma model PLC/PRF/5. Cancer Res. 2006;66:11851.

28. Llovet JM, Ricci S, Mazzaferro V, et al. Sorafenib in advanced hepatocellular carcinoma. $N$ Engl J Med. 2008;359:378-390. doi:10.1056/NEJMoa0708857

29. Sia D, Jiao Y, Martinez-Quetglas I, et al. Identification of an immune-specific class of hepatocellular carcinoma, based on molecular features. Gastroenterology. 2017;153(3):812-826. doi:10.1053/ j.gastro.2017.06.007

30. Cheng AL, Kang Y-K, Chen Z, et al. Efficacy and safety of sorafenib in patients in the Asia-Pacific region with advanced hepatocellular carcinoma: a phase III randomized, double-blind, placebo-controlled trial. Lancet Oncol. 2009;10:25-34.

31. Song H, Liu Q, Liao Q, et al. Circular RNA and tumor microenvironment. Cancer Cell Int. 2020;20:211. doi:10.1186/ s12935-020-01301-z

32. Pompili M, Francica G, Ponziani FR, Iezzi R, Avolio AW. Bridging and downstaging treatments for hepatocellular carcinoma in patients on the waiting list for liver transplantation. World $J$ Gastroenterol. 2013;19:7515-7530. doi:10.3748/wjg.v19.i43.7515

33. Yoo H, Kim JH, Ko G-Y, et al. Sequential transcatheter arterial chemoembolization and portal vein embolization versus portal vein embolization only before major hepatectomy for patients with hepatocellular carcinoma. Ann Surg Oncol. 2011;18:1251-1257. doi:10.1245/s10434-010-1423-3

34. Kallini JR, Gabr A, Salem R, et al. Transarterial radioembolization with Yttrium-90 for the treatment of hepatocellular carcinoma. $A d v$ Ther. 2016;33:699-714. doi:10.1007/s12325-016-0324-7

35. Bouazza F, Poncelet A, Garcia CA, et al. Radioembolisation and portal vein embolization before resection of large hepatocellular carcinoma. World J Gastroenterol. 2015;21:9666. doi:10.3748/wjg. v21.i32.9666

36. Utsunomiya T, Shimada M, Kudo M, et al. A comparison of the surgical outcomes among patients with HBV-positive, HCV-positive, and non-B non-C hepatocellular carcinoma: a nationwide study of 11,950 patients. Ann Surg. 2015;261:513. doi:10.1097/ SLA.000000000000821 
37. Akateh C, Black SM, Conteh L, et al. Neoadjuvant and adjuvant treatment strategies for hepatocellular carcinoma. World $J$ Gastroenterol. 2019;25(28):3704-3721. doi:10.3748/wjg.v25. i28.3704

38. Nault JC, Nahon P. Can we move on from the discussion of direct antiviral agents and risk of hepatocellular carcinoma recurrence? Gastroenterology. 2019;156:1558-1560. doi:10.1053/j. gastro.2019.03.027

39. Huang G, Li -P-P, Lau WY, et al. Antiviral therapy reduces hepatocellular carcinoma recurrence in patients with low HBV-DNA levels: a randomized controlled trial. Ann Surg. 2018;268:943-954. doi:10.1097/SLA.0000000000002727

40. Exposito MJ, Akce M, Alvarez JM, et al. 783TiP CA209-9DX: phase III, randomized, double-blind study of adjuvant nivolumab vs placebo for patients with hepatocellular carcinoma (HCC) at high risk of recurrence after curative resection or ablation. Ann Oncol. 2018;29 (suppl_8).

41. Furtado RV, Ha L, Clarke S, et al. Adjuvant iodine 131Lipiodol after resection of hepatocellular carcinoma. J Oncol. 2015;2015:746917. doi: $10.1155 / 2015 / 746917$

42. Forner A, Reig M, Bruix J. Hepatocellular carcinoma. Lancet. 2018;391(10127):1301-1314.

43. Ikeda M, Mitsunaga S, Ohno I. Systemic chemotherapy for advanced hepatocellular carcinoma: past, present, and future. Diseases. 2015;3:360-381. doi:10.3390/diseases3040360

44. Lai CL, Lok AS-F, Wu P-C, et al. Doxorubicin versus no antitumor therapy ininoperable hepatocellular carcinoma. A prospective randomized trial. Cancer. 1988;62:479-483. doi:10.1002/1097-0142(19880801)62:3<479::AID-CNCR2820620306>3.0.CO;2-L

45. Gish RG, Porta C, Lazar L, et al. Phase III randomized controlled trial comparing the survival of patients with unresectable hepatocellular carcinoma treated with nolatrexed or doxorubicin. J Clin Oncol. 2007;25:3069. doi:10.1200/JCO.2006.08.4046

46. Abdel-Rahman O, Lamarca A. Development of sorafenib-related side effects in patients diagnosed with advanced hepatocellular carcinoma treated with sorafenib: a systematic-review and meta-analysis of the impact on survival. Expert Rev Gastroenterol Hepatol. 2017;11 (1):75-83. doi:10.1080/17474124.2017.1264874

47. Vincenzi B, Santini D, Russo A, et al. Early skin toxicity as a predictive factor for tumor control in hepatocellular carcinoma patients treated with sorafenib. Oncologist. 2010;15:85. doi:10.1634/theoncologist.2009-0143

48. Bruix J, Raoul J-L, Sherman M, et al. Efficacy and safety of sorafenib in patients with advanced hepatocellular carcinoma: subanalyses of a phase III trial. $J$ Hepatol. 2012;57:821. doi:10.1016/j. jhep.2012.06.014

49. Sherman M. Regorafenib for treatment of hepatocellular carcinoma. Hepatology. 2018;67(3):1162-1165. doi:10.1002/hep.29598

50. Kudo M, Finn RS, Qin S, et al. Lenvatinib versus sorafenib in first-line treatment of patients with unresectable hepatocellular carcinoma: a randomised phase 3 non-inferiority trial. Lancet. 2018;391:1163-1173. doi:10.1016/S0140-6736(18)30207-1

51. Cheng A-L, Kang Y-K, Lin D-Y. Sunitinib versus sorafenib in advanced hepatocellular cancer: results of a randomized phase III trial. J Clin Oncol. 2013;31(32):4067-4075. doi:10.1200/ JCO.2012.45.8372

52. Johnson PJI, Qin S, Park J-W, et al. Brivanib versus sorafenib as first-line therapy in patients with unresectable, advanced hepatocellular carcinoma: results from the randomized phase III BRISK-FL study. J Clin Oncol. 2013;31(28):3517-3524. doi:10.1200/ JCO.2012.48.4410

53. Cainap C, Qin S, Huang W-T. Linifanib versus Sorafenib in patients with advanced hepatocellular carcinoma: results of a randomized phase III trial. J Clin Oncol. 2015;33(2):172-179. doi:10.1200/ JCO.2013.54.3298
54. Vilgrain V, Bouattour M, Sibert A, et al. SARAH: a randomized controlled trial comparing efficacy and safety of selective internal radiation therapy (with yttrium-90 microspheres) and sorafenib in patients with locally advanced hepatocellular carcinoma. $J$ Hepatol. 2017;66(1):S85-S86.

55. Asia-Pacific Hepatocellular Carcinoma Trials Group; Chow PKH, Gandhi M, Tan S-B, et al. SIRveNIB: selective internal radiation therapy versus sorafenib in Asia-pacific patients with hepatocellular carcinoma. J Clin Oncol. 2018;36(19):1913-1921. doi:10.1200/ JCO.2017.76.0892.

56. Zhu AX, Rosmorduc O, Evans TRJ, et al. SEARCH: a phase III, randomized, double-blind, placebo-controlled trial of sorafenib plus erlotinib in patients with advanced hepatocellular carcinoma. J Clin Oncol. 2015;33(6):559-566. doi:10.1200/JCO.2013.53.7746

57. Ikeda K, Kudo M, Kawazoe S, et al. Phase 2 study of lenvatinib in patients with advanced hepatocellular carcinoma. $J$ Gastroenterol. 2017;52:512. doi:10.1007/s00535-016-1263-4

58. Cheng AL, Qin S, Ikeda M, et al. IMbrave150: efficacy and safety results from a ph 3 study evaluating atezolizumab (atezo) + bevacizumab (bev) vs sorafenib (sor) as first treatment (tx) for patients (pts) with unresectable Hepatocellular Carcinoma (HCC). Ann Oncol. 2019;30:ix186-ix187.

59. McLellan B, Ciardiello F, Lacouture ME, et al. Regorafenibassociated hand-foot skin reaction: practical advice on diagnosis, prevention, and management. Ann Oncol. 2015;26(10):2017-2026. doi:10.1093/annonc/mdv244

60. Abou-Alfa GK, Meyer T, Cheng A-L, et al. Cabozantinib (C) versus placebo $(\mathrm{P})$ in patients (pts) with advanced hepatocellular carcinoma (HCC) who have received prior sorafenib: results from the randomized phase III CELESTIAL trial. J Clin Oncol. 2018;36(Suppl 4):207. doi:10.1200/JCO.2018.36.4_suppl.207

61. Kelley RK, Verslype C, Cohn AL, et al. Cabozantinib in hepatocellular carcinoma: results of a phase 2 placebo-controlled randomized discontinuation study. Ann Oncol. 2017;28:528. doi:10.1093/annonc/ mdw651

62. Nguyen L, Chapel S, Tran BD, et al. Cabozantinib exposure-response analyses of efficacy and safety in patients with advanced hepatocellular carcinoma. J Pharmacokinet Pharmacodyn. 2019;46 (6):577-589. doi:10.1007/s10928-019-09659-y

63. Zhu AX, Park JO, Ryoo B-Y, et al. Ramucirumab versus placebo as second-line treatment in patients with advanced hepatocellular carcinoma following first-line therapy with sorafenib (REACH): a randomised, double-blind, multicentre, phase 3 trial. Lancet Oncol. 2015;16:859. doi:10.1016/S1470-2045(15)00050-9

64. Zhu AX, Kang YK, Yen CJ, et al. REACH-2 study investigators. Ramucirumab after sorafenib in patients with advanced hepatocellular carcinoma and increased $\alpha$-fetoprotein concentrations (REACH2): a randomised, double-blind, placebo-controlled, phase 3 trial. Lancet Oncol. 2019.

65. Zheng H, Qin Z, Qiu X, Zhan M, Wen F, Xu T. Cost-effectiveness analysis of ramucirumab treatment for patients with hepatocellular carcinoma who progressed on sorafenib with $\alpha$-fetoprotein concentrations of at least $400 \mathrm{ng} / \mathrm{mL}$. J Med Econ. 2020;1-6.

66. Demir T. Systemic therapy of liver cancer. Adv Cancer Res. 2021;149:257-294.

67. El-Khoueiry AB, Sangro B, Yau T, et al. Nivolumab in patients with advanced hepatocellular carcinoma (CheckMate 040): an open-label, non-comparative, Phase $1 / 2$ dose escalation and expansion trial. Lancet. 2017;389(10088):2492-2502. doi:10.1016/S0140-6736(17) 31046-2

68. Zhu AX, Finn RS, Edeline J, et al. Pembrolizumab in patients with advanced hepatocellular carcinoma previously treated with sorafenib (KEYNOTE-224): a non-randomised, open-label phase 2 trial. Lancet Oncol. 2018;19:940-952. doi:10.1016/S1470-2045(18) 30351-6 
69. Finn RS, Ryoo B-Y, Merle P, et al. Pembrolizumab as second-line therapy in patients with advanced hepatocellular carcinoma in KEYNOTE-240: a randomized, double-blind, phase III trial. J Clin Oncol. 2019;38:JCO1901307. doi:10.1200/JCO.19.01307

70. Yau T, Park JW, Finn RS, et al. CheckMate 459: a randomized, multi-center phase III study of nivolumab (NIVO) vs sorafenib (SOR) as first-line (1L) treatment in patients (pts) with advanced hepatocellular carcinoma (aHCC). Ann Oncol. 2019;30(suppl_5): v851-v934. doi:10.1093/annonc/mdz394.029

71. Yau T, Kang Y-K, Kim T-Y, et al. Efficacy and safety of nivolumab plus ipilimumab in patients with advanced hepatocellular carcinoma previously treated with sorafenib: the checkmate 040 randomized clinical trial. JAMA Oncol. 2020;6(11):e204564. doi:10.1001/jamaoncol.2020.4564

72. Tsang J, Wong JSL, Kwok GGW, et al. Nivolumab + ipilimumab for patients with hepatocellular carcinoma previously treated with sorafenib. Expert Rev Gastroenterol Hepatol. 2021;1-10. Doi:10.1080/17474124.2021.1899808

73. Gordan JD, Kennedy EB, Abou-Alfa GK, et al. Systemic therapy for advanced hepatocellular carcinoma: ASCO guideline. J Clin Oncol. 2020;38:JCO2002672. doi:10.1200/JCO.20.02672

74. Heimbach JK, Kulik LM, Finn RS, et al. AASLD guidelines for the treatment of hepatocellular carcinoma. Hepatology. 2018;67 (1):358-380. doi:10.1002/hep.29086

75. Abou-Alfa GK, Meyer T, Cheng A-L, et al. Cabozantinib in patients with advanced and progressing hepatocellular carcinoma. $N$ Engl J Med. 2018;379:54. doi:10.1056/NEJMoa1717002

76. Turkes F, Chau I. Ramucirumab and its use in the treatment of hepatocellular carcinoma. Future Oncol. 2019;15(9):979-988. doi:10.2217/fon-2018-0822

77. Cidon EU. Systemic treatment of hepatocellular carcinoma: past, present and future. World $J$ Hepatol. 2017;9(18):797-807. doi:10.4254/wjh.v9.i18.797

78. Sim HW, Knox J. Hepatocellular carcinoma in the era of immunotherapy. Curr Probl Cancer. 2018;42(1):40-48. doi:10.1016/j.currproblcancer.2017.10.007

79. Mahipal A, Tella SH, Kommalapati A, et al. Immunotherapy in hepatocellular carcinoma: is there a light at the end of the tunnel? Cancers (Basel). 2019;11(8):1078. doi:10.3390/cancers11081078

80. Yau T, Kang Y-K, Kim T-Y, et al. Nivolumab (NIVO) + ipilimumab (IPI) combination therapy in patients (pts) with advanced hepatocellular carcinoma (aHCC): results from CheckMate 040. J Clin Oncol. 2019;37:4012. doi:10.1200/JCO.2019.37.15_suppl.4012

81. Lee WS, Yang H, Chon HJ, et al. Combination of anti-angiogenic therapy and immune checkpoint blockade normalizes vascular-immune crosstalk to potentiate cancer immunity. Exp Mol Med. 2020;52(9):1475-1485. doi:10.1038/s12276-020-00500-y

82. Kudo M. Recent advances in systemic therapy for hepatocellular carcinoma in an aging society: 2020 update. Liver Cancer. 2020;9 (6):640-662. doi:10.1159/000511001

83. Park R, Eshrat F, Al-Jumayli M, Saeed A, Saeed A. Immunooncotherapeutic approaches in advanced hepatocellular carcinoma. Vaccines (Basel). 2020;8(3):447. doi:10.3390/vaccines 8030447

84. Aitcheson G, Pillai A, Dahman B, John BV. Recent advances in systemic therapies for advanced hepatocellular carcinoma. Curr Hepatol Rep. 2021;1-11. doi:10.1007/s11901-021-00560-2
85. Ikeda M, Sung MW, Kudo M, et al. A phase $1 \mathrm{~b}$ trial of lenvatinib plus pembrolizumab in patients with unresectable hepatocellular carcinoma. JCO. 2018;36:4076. doi:10.1200/JCO.2018.36.15_suppl.4076

86. Yau T, Rimassa L, Cheng A-L, et al. Phase 3 (COSMIC-312) study of cabozantinib (C) in combination with atezolizumab (A) versus sorafenib (S) in patients (pts) with advanced hepatocellular carcinoma (aHCC) who have not received previous systemic anticancer therapy. Ann Oncol. 2019;30(suppl_9):ix42-ix67. doi:10.1093/annonc/ mdz422.075

87. Abou-Alfa GK, Chan SL, Furuse J, et al. A randomized, multicenter phase 3 study of durvalumab (D) and tremelimumab (T) as first-line treatment in patients with unresectable hepatocellular carcinoma (HCC): HIMALAYA study. J Clin Oncol. 2018;36:TPS4144TPS4144. doi:10.1200/JCO.2018.36.15_suppl.TPS4144

88. Floudas C, Xie C, Brar G, et al. Combined immune checkpoint inhibition with tremelimumab and durvalumab in patients with advanced hepatocellular carcinoma or biliary tract carcinomas. J Clin Oncol. 2019;37(4_suppl):336. doi:10.1200/JCO.2019.37.4_suppl.336

89. Tai D, Choo SP, Chew V. Rationale of immunotherapy in hepatocellular carcinoma and its potential biomarkers. Cancers (Basel). 2019;11(12):1926. doi:10.3390/cancers11121926

90. Finn RS, Ikeda M, Zhu AX, et al. Phase Ib study of lenvatinib plus pembrolizumab in patients with unresectable hepatocellular carcinoma. J Clin Oncol. 2020;38(26):2960-2970. doi:10.1200/ JCO.20.00808

91. Havel JJ, Chowell D, Chan TA. The evolving landscape of biomarkers for checkpoint inhibitor immunotherapy. Nat Rev Cancer. 2019;19:133-150.

92. Kaseb AO, Vence L, Blando J, et al. Immunologic correlates of pathologic complete response to preoperative immunotherapy in hepatocellular carcinoma. Cancer Immunol Res. 2019;7:1390-1395. doi:10.1158/2326-6066.CIR-18-0605

93. Khagi Y, Goodman AM, Daniels GA, et al. Hypermutated circulating tumor DNA: correlation with response to checkpoint inhibitor-based immunotherapy. Clin Cancer Res. 2017;23:5729-5736. doi:10.1158/ 1078-0432.CCR-17-1439

94. Moeini A, Torrecilla S, Tovar V, et al. An immune gene expression signature associated with development of human hepatocellular carcinoma identifies mice that respond to chemopreventive agents. Gastroenterology. 2019;157(5):1383-1397.e11. doi:10.1053/j. gastro.2019.07.028

95. Chew SA, Moscato S, George S, et al. Liver cancer: current and future trends using biomaterials. Cancers (Basel). 2019;11(12): E2026. doi:10.3390/cancers11122026

96. Zhao Y, Fan Z, Shen M, et al. Capturing hepatocellular carcinoma cells using lactobionic acid-functionalized electrospun polyvinyl alcohol/polyethyleneimine nanofibers. RSC Adv. 2015;5:70439-70447. doi:10.1039/C5RA11662G

97. Broutier L, Mastrogiovanni G, Verstegen MM, et al. Human primary liver cancer-derived organoid cultures for disease modeling and drug screening. Nat Med. 2017;23:1424-1435. doi:10.1038/nm.4438

\section{Publish your work in this journal}

The Journal of Hepatocellular Carcinoma is an international, peerreviewed, open access journal that offers a platform for the dissemination and study of clinical, translational and basic research findings in this rapidly developing field. Development in areas including, but not limited to, epidemiology, vaccination, hepatitis therapy, pathology and molecular tumor classification and prognostication are all considered for publication. The manuscript management system is completely online and includes a very quick and fair peer-review system, which is all easy to use. Visit http://www.dovepress.com/ testimonials.php to read real quotes from published authors. 